05

\title{
Моделирование перехода графита в алмаз в изэнтропическом процессе
}

\author{
(C) B.B. Прут \\ Московский фризико-технический институт, \\ 141700 Долгопрудный, Московская область, Россия \\ e-mail: vvprut@gmail.com
}

(Поступило в Редакцию 3 ноября 2015 г.)

Построено уравнение состояния графита и алмаза в широком диапазоне плотностей и температур. Приведена система уравнений фазового перехода графита в алмаз. Вычислены ударные адиабаты графита и алмаза. Изложены результаты численного моделирования превращения графита в алмаз в изэнтропическом процессе сжатия в металлическом z-пинче с сохранением алмаза.

DOI: 10.21883/JTF.2017.05.44442.1641

\section{Введение}

Уникальные свойства алмаза стимулировали исследования и поиски его применений во многих областях, в том числе в оптике, оптоэлектронике, электронике, биологии и электрохимии. Превращение графита в алмаз реализуется статическими и динамическими методами. Статический синтез производят в области фазовой диаграммы углерода, где алмаз термодинамически более устойчив графита. Используется каталитический метод при давлении $P \sim 40-100 \mathrm{kbar}$, температурах $T \sim(1-2.5) \cdot 10^{3} \mathrm{~K}$ и металлах в качестве растворителей - катализаторах. Можно получить алмазы с характерными размерами $\sim 0.1-10 \mathrm{~mm}[1,2]$.

Применяются также иные статические методы. Методом парофазного химического осаждения можно выращивать поликристаллические алмазные пластины диаметром более $200 \mathrm{~mm}$. Для большинства применений алмаза в современной технологии требуются монокристаллические алмазные подложки или пленки больших размеров (25 mm и более), пригодные для фотолитографического процесса. До сих пор размер остается главной проблемой, поскольку в настоящее время доступны кристаллы алмаза размером не более $10 \mathrm{~mm}$ [3].

В качестве динамического метода используется ударно-волновое сжатие, создаваемое взрывчатыми веществами. Характерные параметры ударных волн в углероде $P \sim 300 \mathrm{kbar}, T \sim(1-3) \cdot 10^{3} \mathrm{~K}$. Искусственный алмаз получается в виде порошка с размером зерна $\sim 10 \mu \mathrm{m}[4]$. Эти методы дают алмазы не ювелирного качества с примесями. Предлагались также другие способы получения искусственных алмазов [5].

Ни один из существующих и предложенных методов не оптимален. Поэтому научный и практический интерес представляет рассмотрение альтернативных методов. Кроме того, природа происхождения алмаза, несмотря на различные гипотезы, также требует детального изучения, для чего необходимо создание соответствующих давлений и температур.

Преимуществом метода металлического $z$-пинча по сравнению с взрывными методами изэнтропического сжатия является возможность сохранения образца. Цель настоящей работы показать возможность перехода графита в алмаз с сохранением алмаза в контейнере. Для осуществления этой цели проведено численное моделирование превращения графита в алмаз в изэнтропическом процессе в металлическом $z$-пинче. Построено уравнение состояния графита и алмаза в широком диапазоне плотностей и температур. Предложено математическое описание системы уравнений фазового перехода графит-алмаз. Проведено сравнение с экспериментальными ударными адиабатами.

\section{Постановка задачи}

Сжатие вещества осуществляется в металлическом $z$-пинче аналогично [6-8]. Блок коаксиальных цилиндров состоит из трех веществ. Внутреннего - графита. Внешнего - вещества с хорошей проводимостью: меди или алюминия. По внешнему цилиндру протекает ток конденсаторной батареи величиной $\sim 10$ МА с характерным временем $\sim 10 \mu \mathrm{s}$, создающий магнитное поле и давление $\sim 0.1-1 \mathrm{Mbar}$, которое сжимает все вещества. Джоулев нагрев нагревает внешнюю трубку до высоких температур, однако из-за скинирования тока за время процесса ток не успевает проникнуть во внутреннюю часть трубки и нагреть ни средний цилиндр, ни графит. Если ток достаточно велик, наружная часть токонесущей трубки взрывается, однако средняя трубка служит контейнером, в котором сохраняется углерод.

В квазистатических условиях максимальное сжатие достигается в первом полупериоде тока, весь дальнейший ток является „паразитическим“. Он вызывает лишь нагрев и взрыв устройства, поэтому для сохранения образца должен быть выключен. Это достигается введением в электрическую цепь переменного электрического сопротивления - аналога плавкого предохранителя. Его параметры выбираются таким образом, чтобы максимально достижимое давление снижалось несущественно, но после максимально достигнутого давления этот предохранитель резко увеличивал свое сопротивление и 
диссипировал всю оставшуюся энергию в конденсаторной батарее и магнитном поле.

Многочисленные эксперименты показали [6-8], что процесс сжатия в значительной степени одномерный. Поэтому для моделирования использовалась система одномерных уравнений магнитной гидродинамики в лагранжевом представлении и цилиндрической симметрии [9].

Безразмерное уравнение электротехнической цепи

$$
\frac{d(1+\Delta L) J}{d t}+\beta_{L} E_{j}=\beta_{c} U, \quad \frac{d U}{d t}=-\beta_{c} J,
$$

где $U$ - напряжение на конденсаторной батарее, $J$ ток в цепи, безразмерные определяющие параметры: $\beta_{c}=10 R_{0}^{2}\left(\pi \rho_{0}\right)^{1 / 2} \omega_{0} / J_{0}, \beta_{L}=2 \cdot 10^{-9} l / L_{0}, \omega_{0}$ - начальная частота, $L_{0}, \Delta L-$ начальная и дополнительная (безразмерная к $L_{0}$ ) индуктивности, $l$ - длина трубки.

Граничные условия на свободной внешней поверхности проводника радиуса $R: \sigma_{r}=0, \lambda \frac{\partial T}{\partial r}=2 \sigma_{S B} T^{4}$, $H=\frac{0.2 J}{R}$; на оси $u=0, \frac{\partial T}{\partial r}=0, H=0 . \sigma_{S B}$ - постоянная Стефана-Больцмана.

Начальные условия: $T=300 \mathrm{~K}, p=1 \mathrm{bar}, u=0, J=0$, $U=U_{0}$. Задача численно решалась по разностной схеме, в которой сетка могла быть неоднородной и сгущаться как к оси, так и границе. Количество точек по $r$ до 1500 , тогда количество точек по $t$, которое определялось исходя из условий устойчивости и точности, до $\sim 10^{5}$.

\section{Уравнение состояния, электропроводность и теплопроводность меди}

В приводимом здесь варианте расчетов в качестве проводящего вещества использовалась медь. Его уравнение состояния (кроме „холодной“ составляющей) принято в виде [10], которое дает приемлемую точность во всей нерелятивистской области параметров.

Электропроводность $\sigma$ во всем диапазоне рассматриваемых параметров интерполирована между электропроводностями твердого тела $\sigma_{s}$ и идеальной плазмы $\sigma_{p}$ : $\sigma_{s}=\left(\sigma_{s}^{\xi}+\sigma_{p}^{\xi}\right)^{1 / \xi}$, где параметр $\xi$ варьировал $\xi=1-2$. Примем $\sigma_{s}=\operatorname{const}(z / \varphi)$, где функция $\varphi$ определяется экспериментальными значениями и учитывает зависимость электрического сопротивления от температуры $\varphi \sim T^{5}$ при низких температурах, $\varphi \sim T$ в области нормальных температур, и скачок при плавлении. Она также учитывает зависимость от плотности в соответствии с [11]. Проводимость плазмы $\sigma_{p}=e^{2} n_{e} / v, v=v_{i e}+v_{a}$, где $v_{i e}$ - частота столкновений с ионами и электронами, $v_{a}$ - частота столкновений с атомами, тем самым это соотношение позволяет аппроксимировать также и частично ионизованную плазму. Теплопроводность представлена аддитивно $\lambda=\lambda_{e}+\lambda_{h}$, где $\lambda_{e}-$ электронная теплопроводность и $\lambda_{h}$ - лучистая теплопроводность. Анализ процессов переноса излучения показывает, что в рассматриваемом диапазоне параметров можно ограничиться приближением лучистой теплопроводности и нет необходимости рассматривать неравновесный перенос излучения [12]. Электронная теплопроводность $\lambda_{e}$ вычислялась исходя из соотношения Видемана-Франца $\lambda_{e}=L \sigma T$, где $L-$ константа Лоренца, которая для твердого тела $\left(L_{s}=\pi^{2} / 3 e^{2}\right)$ и плазмы близка $\left(L_{s}=3 / e^{2}\right)$. Лучистая теплопроводность вычислялась по формуле $\lambda_{h}=(16 / 3) \sigma_{S B} l_{R} T^{3}$, где $l_{R}=\mathrm{const} V^{2} T^{7 / 2} /\left(z(z+1)^{2}\right)-$ средняя росселандова длина пробега [13].

\section{Уравнение состояния графита и алмаза}

Примем для холодной энергии углерода следующую форму во всем диапазоне плотностей: нерелятивистской и релятивистской.

При $0<x \leq \sim 10$

$$
E_{x}=E_{F} \times\left(1-x^{\alpha}\right)^{2} e^{-b x^{\beta}},
$$

где $E_{F}=E_{F, 0} /\left(x^{2 / 3}+r_{0} x^{1 / 3}\right), b=\ln \left(\tilde{E}_{F, 0} 2 \alpha^{2} /\left(B_{0} V_{0} x^{\chi}\right)\right)$.

Здесь и ниже $V$ - удельный объем $\left(\mathrm{cm}^{3} / \mathrm{mol}\right)$, $x=V / V_{0}$ - относительный удельный объем, $E-$ энергия $(\mathrm{J} / \mathrm{mol})$. Индекс „, $0^{\star}$ относится к условиям $T=0$, $P=0$. Индекс „1“ относится к „нормальным“ условиям $T_{1}=298 \mathrm{~K}, P_{1}=1$ bar.

Первый множитель в формуле (1) определяет энергию идеального вырожденного нерелятивистского и однородного релятивистского электронного газа.

$E_{F, n r}=\frac{3}{10}\left(3 \pi^{2}\right)^{2 / 3} \frac{\hbar^{2}}{m_{e}} \frac{Z^{5 / 3}}{V^{2 / 3}} N_{A}-$ энергия идеального однородного вырожденного нерелятивистского электронного газа, $Z$ - атомный номер, $N_{A}$ - число Авогадро. $\quad E_{F, r}=\frac{3}{4}\left(3 \pi^{2}\right)^{1 / 3} \hbar c \frac{Z^{4 / 3}}{V^{1 / 3}} N_{A}-\quad$ энергия идеального однородного вырожденного релятивистского электронного газа. Комбинация обеих энергий в вышеприведенном виде, где $E_{F, 0}=E_{F, n r}\left(V_{0}\right), \tilde{E}_{F, 0}=$ $=E_{F, 0}\left(1+c_{0}\right), \quad E_{F, 0}=1.505397 \cdot 10^{6} Z^{5 / 3} / V_{0}^{2 / 3} \mathrm{~J} / \mathrm{mol}$, $E_{F, n r}=E_{F, 0} / x^{2 / 3}$, безразмерный коэффициент $r_{0}=$ $=6 / 5\left(3 \pi^{2}\right)^{1 / 3} \lambda_{C} Z^{1 / 3}\left(V_{0} / N_{A}\right)^{-1 / 3}, \quad \lambda_{C}=\hbar / m_{e} c$ комптоновская длина волны, позволяет получить простую формулу во всем диапазоне плотностей $[14,15]$.

Второй множитель в формуле (1) необходим для того, чтобы автоматически выполнить условия $E\left(V=V_{0}\right)=0$, давление $P\left(V=V_{0}\right)=0$, а следующие производные энергии $\neq 0$.

Третий множитель в формуле (1) сконструирован так, что модуль сжатия $B\left(V_{0}\right)=B_{0}$ определяется автоматически. Однако параметр $q=-\frac{\partial \ln B}{\partial \ln V}$ определяется численным решением уравнения $q\left(V_{0}\right)=q_{0}$. В это уравнение входят три свободных параметра: $\alpha, \beta, \chi$. Численный анализ уравнения состояния показал, что оказываются удовлетворительными значения $\alpha=1 / 3, \beta=1 / 3$. Тогда параметр $q$ вычисляется из простого однопараметрического уравнения относительно $\chi$.

Конечно, учет релятивистских эффектов лежит далеко за пределами необходимой области плотностей решаемой здесь задачи. Однако формула (1) иллюстрирует 

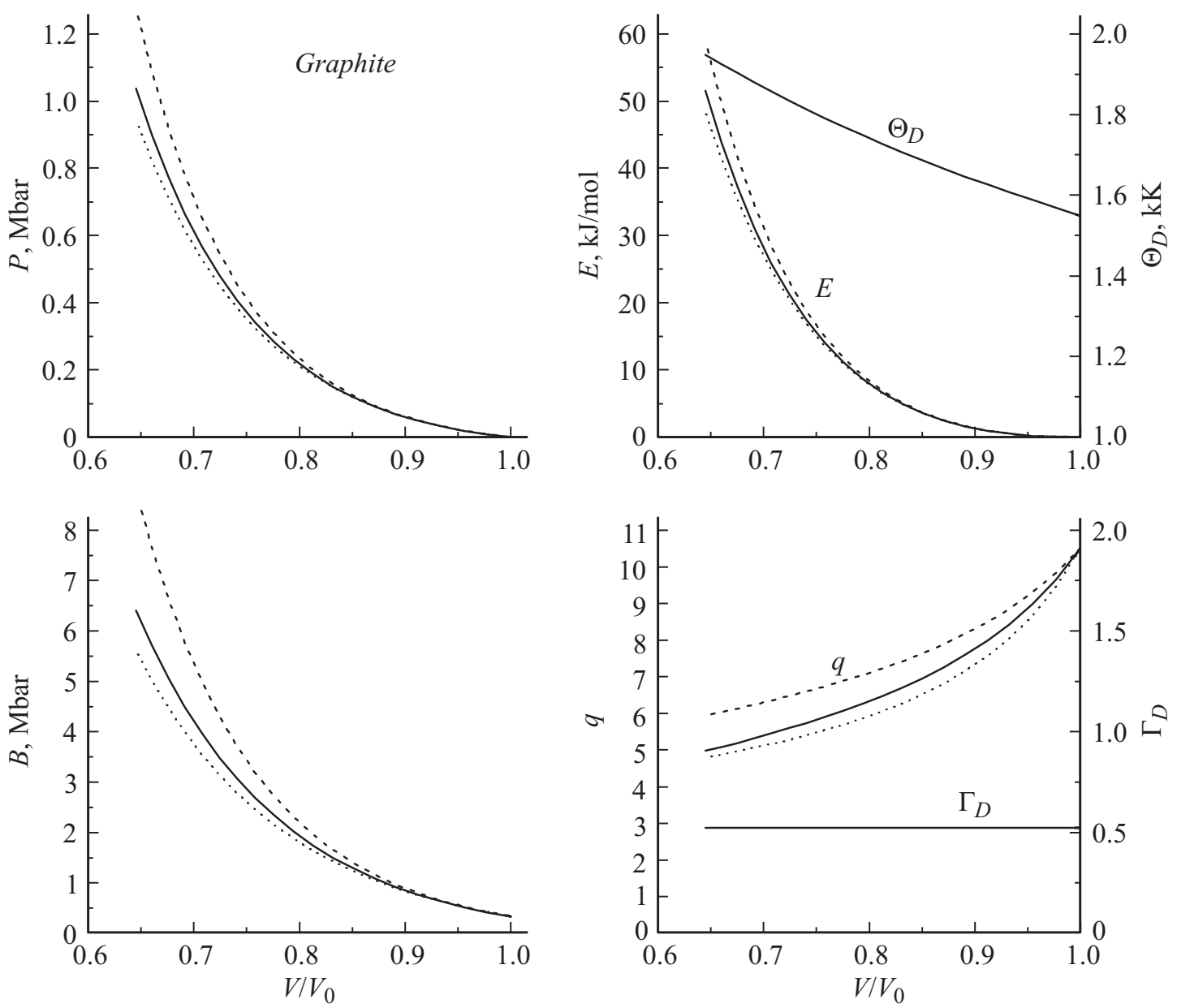

Рис. 1. Зависимости для графита энергии, давления, модуля сжатия, параметра $q$, температуры Дебая, параметра Грюнайзена от относительной плотности до давления $\sim 1$ Mbar. Штриховыми линиями показаны зависимости по формулам Ванье, точечными линиями - по формулам Мурнагана-Берча.

возможности метода и может оказаться полезной при моделировании структуры планет и звезд.

Параметры графита определялись в экспериментальных (статических и ударно-волновых) [16-27] и теоретических [28-31] работах. Для графита приняты следующие значения: $V_{0}=5.286 \mathrm{~cm}^{3} / \mathrm{mol}$ $\left(\rho_{0}=2.266 \mathrm{~g} / \mathrm{cm}^{3}\right), \quad E_{\text {bond }, 0}=0 \mathrm{~J} / \mathrm{mol}, \quad B_{S, 0}=336 \mathrm{kbar}$, $q_{1}=10.5, \Theta_{D, 0}=1550 \mathrm{~K}$ и $\Gamma_{D, 0}=0.52$. Вычисленные зависимости энергии, давления, модуля сжатия и параметра q, температуры Дебая и параметра Грюнайзена при $T=0$ показаны на рис. 1 до $P \approx 1$ Mbar. Там же дано сравнение с уравнениями Мурнагана-Берча [32] и Ванье [33], наиболее часто используемые для обработки экспериментальных и теоретических данных.

Параметры алмаза определялись в экспериментальных [34-41] и теоретических [42-47] работах. Для алмаза приняты следующие значения: $V_{0}=3.417 \mathrm{~cm}^{3} / \mathrm{mol}$ $\left(\rho_{0}=3.5155 \mathrm{~g} / \mathrm{cm}^{3}\right), \Delta E_{0}=1.82 \cdot 10^{3} \mathrm{~J} / \mathrm{mol}$ относительно графита, $B_{S, 0}=4.46 \mathrm{Mbar}, q_{0}=4.0, \Theta_{D, 0}=1874 \mathrm{~K}$ и $\Gamma_{D, 0}=1.0$. Вычисленные зависимости энергии, давления, модуля сжатия и параметра $q$ при $T=0$ показаны на рис. 2 до $P \approx 10 \mathrm{Mbar}$, а на рис. 3 - до относительной плотности $10^{-8}$, где приведено также сравнение с квазиклассическим уравнением состояния [48,49].

Принятые здесь для алмаза величины $B_{0}$, по-видимому, надежно определены как в экспериментальных, так и теоретических работах. Разброс значений $\Delta B / B<\sim 1 \%$. Совершенно иная ситуация с параметром $q$. По-видимому, наиболее вероятный диапазон $3<q<4[46]$.

Приведенное сравнение показывает, что наши зависимости совпадают с уравнениями Мурнагана-Берча и Ванье для графита и алмаза лишь в достаточно узкой области плотностей, несмотря на то что все они построены для одних и тех же экспериментальных данных: $V_{0}, B_{0}, q_{0}$. Наши расчеты лежат между ними. Относительное расслоение увеличивается для функций, обладающих большим порядком производной энергии по объему. Для алмаза расхождение между этими зависимостями начинается при значительно более высоких давлениях. Функции Мурнагана-Берча и Ванье обладают неправильной асимптотикой, поэтому их нельзя применять при относительном сжатии $V<\sim 0.7 V_{0}$, где 

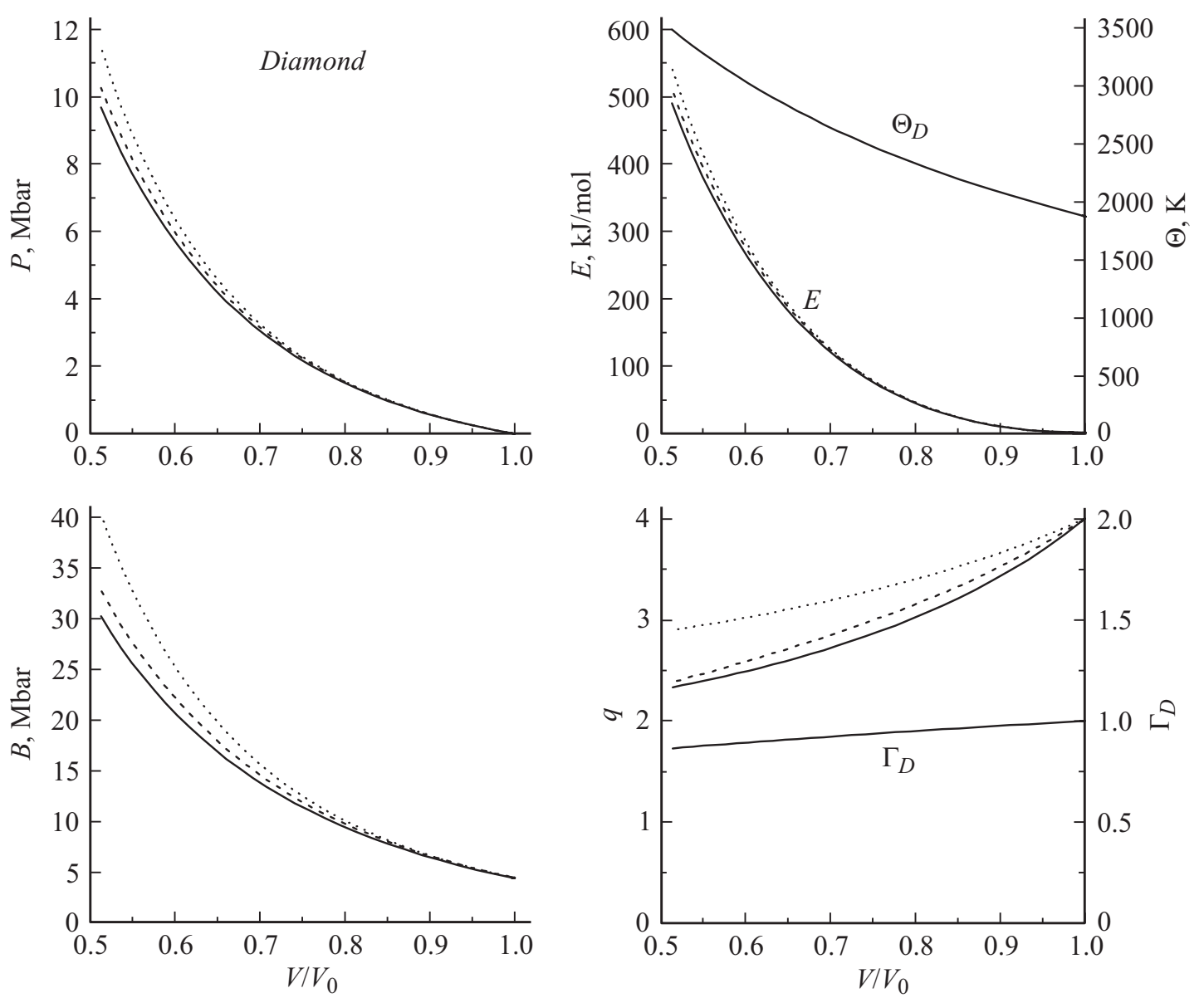

Рис. 2. Зависимости, приведенные на рис. 1 , для алмаза до давления $\sim 10 \mathrm{Mbar}$.

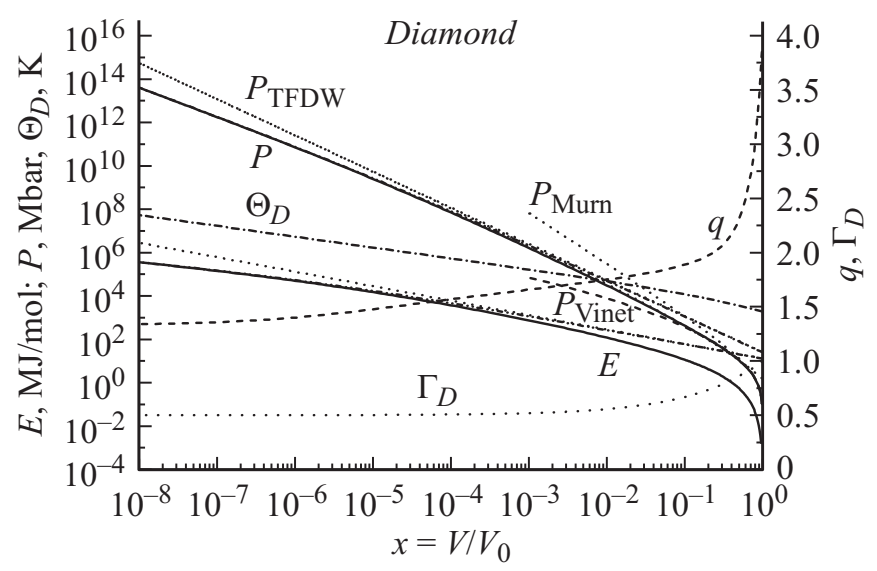

Рис. 3. Зависимости для алмаза энергии, давления, параметpa $q$, температуры Дебая, параметра Грюнайзена до относительной плотности $V / V_{0}=10^{-8}$.

относительная величина погрешности, по-видимому, начинает превышать 0.1 .

Поскольку тепловые составляющие свободной энергии приняты по [10], энтропия и вторые производные свободной энергии (теплоемкость и коэффициент теплового расширения) совпадают с экспериментальными значениями с точностью принятых значений температуры Дебая и параметра Грюнайзена.

\section{Фазовый переход}

Фазовый переход графит-алмаз опишем системой уравнений:

$$
\begin{gathered}
T_{g}=T_{d}=T, \\
P_{g}\left(V_{g}, T\right)=P_{d}\left(V_{d}, T\right), \\
V=k_{g} V_{g}+k_{d} V_{d}, \\
S=k_{g} S_{g}+k_{d} S_{d},
\end{gathered}
$$

где $0 \leq k_{g}, k_{d} \leq 1, k_{g}, k_{d}=1-k_{g}-$ концентрации графита и алмаза. Индекс „g“ относится к графиту, индекс , $d^{\text {“ }}$ - к алмазу.

Однако второе условие фазового равновесия $G_{g}=$ $=G_{d}$, вообще говоря, не выполняется. Здесь $G=E-T S+P V-$ энергия Гиббса, $E=k_{g} E_{g}+k_{d} E_{d}$.

Рассматривались два способа определения $k_{g}$. В первом способе определялось кинетическое уравнение в виде

$$
\frac{d k_{g}}{d t}=\frac{k_{d}}{\tau_{g d}}\left(\left(V_{b} / V\right)^{\alpha}-1\right),
$$

где $\tau_{g d}$ - характерное время перехода, $\alpha-$ параметр. 
Во втором способе кинетика не рассматривалась, а концентрация определялась только как функция удельного объема

$$
k_{g}=\Phi(V)
$$

при $V_{c}<V<V_{b}$. При $V \geq V_{b} k_{g}=1$, при $V \leq V_{c} k_{g}=0$. $V_{b}$ и $V_{c}$ - удельные объемы начала и конца перехода. Вид функции и значения параметров ее определяющих вычислялись из сравнения с экспериментальными данными и приведены ниже. Как видно, в обеих формулах (3) и (4) нет зависимости от температуры, хотя экспериментально в ударных волнах ее влияние в определенной степени заметно. Однако выделить действие температуры количественно, по-видимому, не представляется возможным на основе известных экспериментальных данных.

Объемный модуль сжатия в двухфазной области вычислялся по формуле $B=V /\left(k_{g} V_{g} / B_{g}+k_{d} V_{d} / B_{d}\right)$. Скорость звука $B_{s}=\rho c_{s}^{2}$.

Для определения в двухфазной области $V_{g}, V_{d}$ и $T$ при вычисленном из гидродинамических уравнений удельном объеме $V$ и энтропии $S$ совместно с (3) или (4) решались уравнения (2).

\section{Ударная адиабата графита и алмаза}

Были вычислены ударные адиабаты сплошного графита и сплошного и пористого алмаза. На рис. 4 представлены наши численные расчеты в сравнении с экспериментальными результатами [25,27,39]. Среди опубликованных экспериментальных ударных адиабат углерода изложение результатов в [25], по-видимому, наиболее представительно. Следует заметить, что все опубликованные ударные адиабаты графита даже для одного его типа заметно различаются. Обычно это связывается с различной структурой графита. Однако анализ этих различий выходит за рамки настоящей работы. Для вычисления ударной адиабаты углерода в двухфазной области использовалось только уравнение (4). Из сравнения с экспериментальными результатами [25] определялась функция уравнения (4) и параметры $V_{b}$ и $V_{c}$.

Как видно из сравнения с „алмазными“ адиабатами, очевидна несогласованность численных и экспериментальных результатов [39]. Существует большое расхождение между расчетной и экспериментальной адиабатой сплошного алмаза [39]. Это ожидаемый результат, поскольку вычисленная по $B=\rho_{0} U_{S, 0}^{2}$ величина $B=5.16 \mathrm{Mbar}$, что составляет $\Delta B / B \approx 15 \%$ и лежит далеко за пределами обычной экспериментальной ошибки. В то же время для пористого алмаза получено вполне удовлетворительное согласие с [39] (на рис. 4 они практически неотличимы).

Несколько необычен результат для ударной адиабаты графита. Как видно из рис. 4, параметр $q$ резко падает
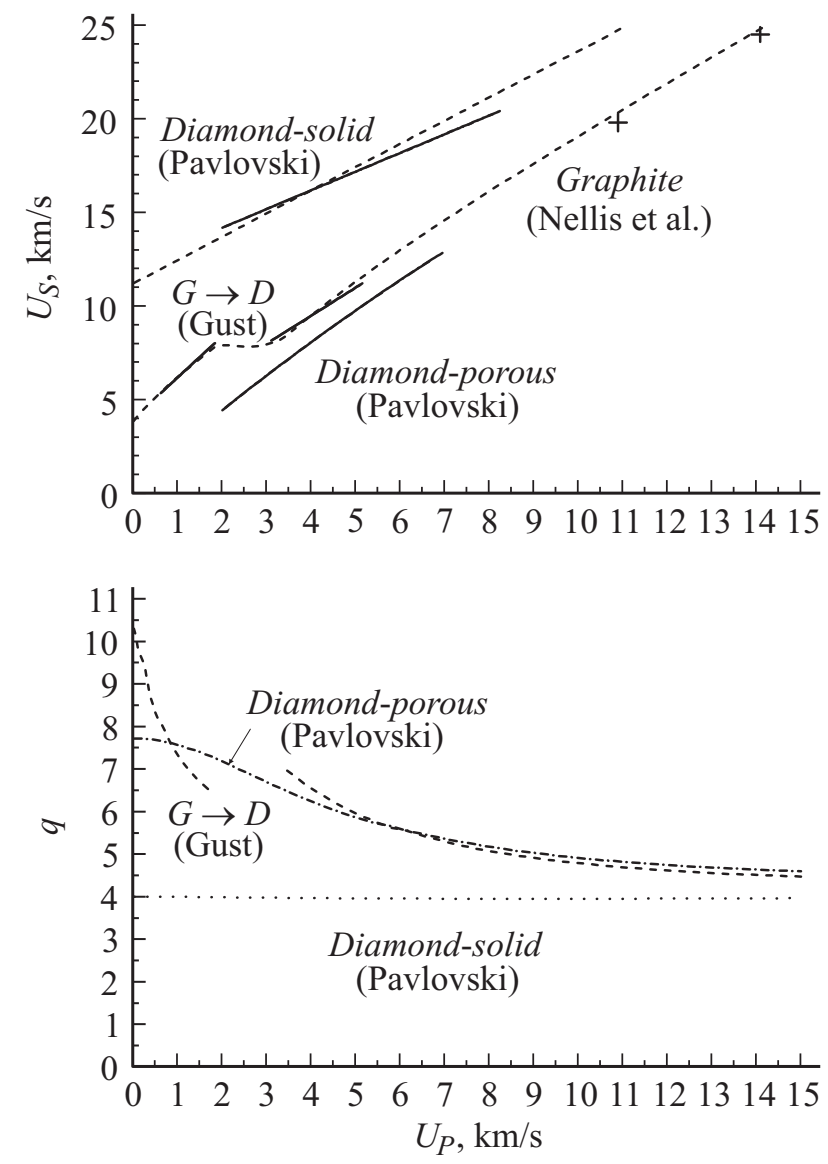

Рис. 4. Расчеты в сравнении с экспериментальными результатами $[25,27,39]$ для графита и алмаза в зависимости от массовой скорости.

при сжатии. Поэтому, чтобы согласовать с экспериментальной адиабатой [25], необходимо увеличить среднее значение $q \approx 8$, полученное в диапазоне скоростей $0.6<U_{p}<1.9$ [25], до величины $q=10.5$.

На рис. 5 показаны зависимости в области перехода, где значительно увеличиваются плотность и температура. Однако температура Дебая и параметр Грюнайзена изменяются очень мало. Естественно, увеличивается энтропия, а также полное давление и энергия. Холодные и тепловые составляющие давления и энергии для каждой из фаз увеличиваются, однако из-за „игры“ величины концентрации холодная и тепловая составляющие графито-алмазной смеси давления увеличиваются, тепловая составляющая энергии увеличивается, а холодная - уменьшается. Однако полная энергия Гиббса, естественно, уменьшается, поскольку происходит переход в более стабильную фазу. Холодные составляющие давления и энергии для каждой из фаз увеличиваются, а тепловые составляющие, меньшие нуля, уменьшаются. Теплоемкость, значение которой в нормальных условиях из-за большой температуры Дебая гораздо меньше классического, вначале перехода уже имеет величину $c_{V} \approx 3 R$. 

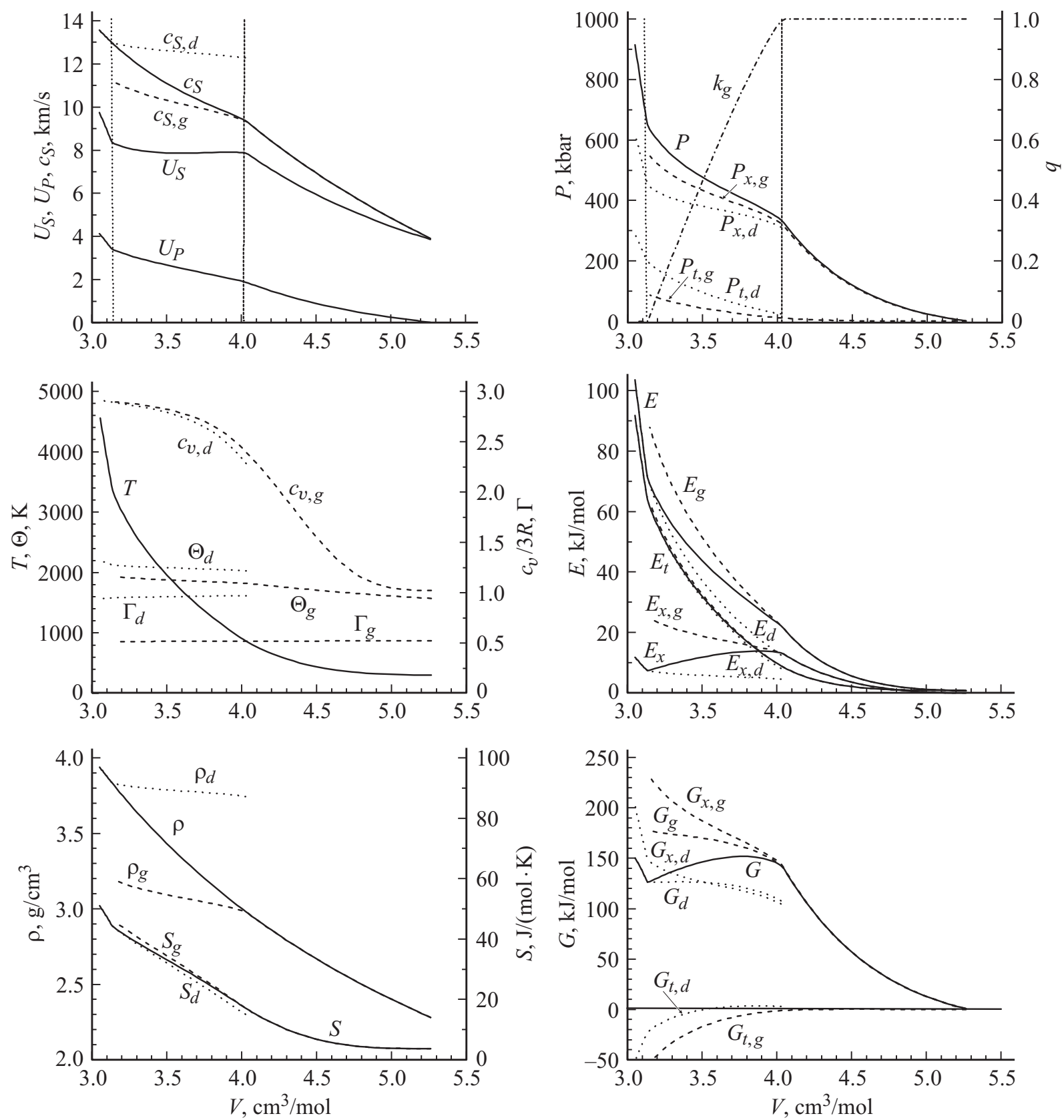

Рис. 5. Расчеты для ударной адиабаты в области перехода графит-алмаз в зависимости от объема.

\section{Изэнтропическое сжатие углерода}

Чтобы продемонстрировать возможности предлагаемого метода, решение магнитогидродинамических уравнений представлено со следующими параметрами. Электротехнические параметры: ток $J_{0}=10 \mathrm{MA}, \omega_{0}=$ $=3.14 \cdot 10^{5} \mathrm{~s}^{-1} \quad(T / 4=5 \mu \mathrm{s})$, энергия конденсаторной батареи $W_{c}=0.75 \mathrm{MJ}$. Радиусы трубок: $R_{1}=0.35 \mathrm{~cm}$, $R_{2}=0.4 \mathrm{~cm}, \quad R_{3}=0.75 \mathrm{~cm}$. Магнитное поле $H_{0}=2.7 \mathrm{MG}$, давление магнитного поля $P_{H^{2}, 0}=$ $=h^{2} / 8 \pi=283 \mathrm{kbar}$.

Параметры внешнего сопротивления $R_{\text {ext }}$ (электропроводность, сечение, толщина и длина фольги) вы- бираются таким образом, чтобы вначале оно было относительно мало и резко (экспоненциально) нарастало после максимального сжатия. Начальное сопротивление принято $R_{\text {ext }} / R_{w}=2 \cdot 10^{-3}$, где $R_{w}$ - волновое сопротивление электрической цепи.

Радиус медной трубки выбирается из следующего компромисса. Давление магнитного поля лежит в необходимом диапазоне, токовая волна за время сжатия не доходит до внутренней границы трубки, а внешняя граница трубки не успевает существенно увеличиться. В этом варианте расчетов внешняя часть трубки разлетается свободно, однако оптимальным может оказаться разлет в некую среду. 

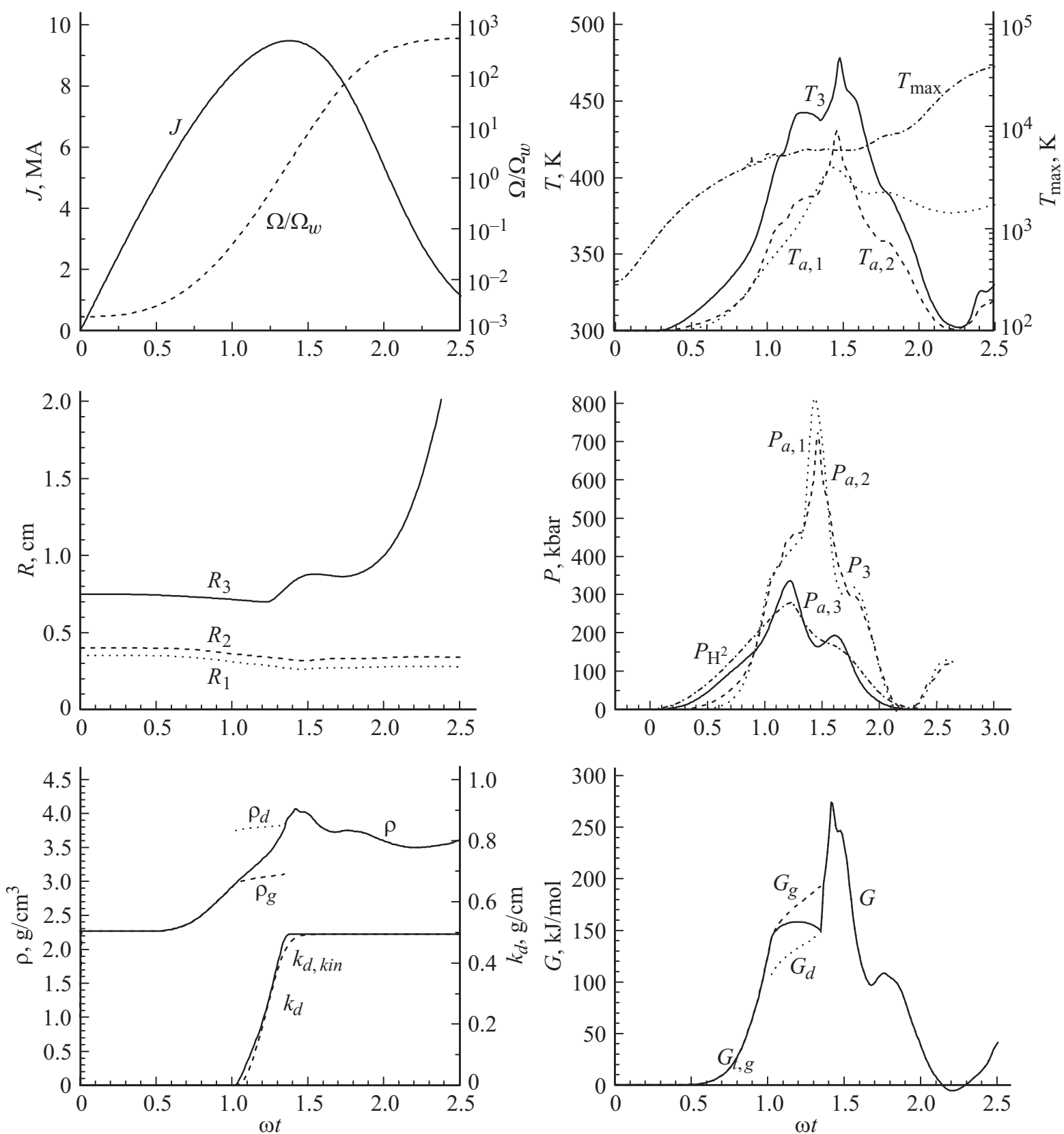

Рис. 6. Временные зависимости тока, сопротивления, радиусов, плотности, температуры, давления и энергии Гиббса.

Материал промежуточной трубки предпочтительно должен обладать сравнительно высокой температурой плавления и малой теплопроводностью. Однако, как в представленном варианте, это не обязательное требование и определяется другими параметрами. Здесь материал промежуточной трубки - вольфрам.

На рис. 6 представлены временные зависимости тока, сопротивления, радиусов, плотности, температуры, давления и энергии Гиббса.

Выбранные параметры сопротивления незначительно уменьшают максимальный ток: $J_{\max }=0.94$, соответственно максимальное давление магнитного поля $P_{B, \max } / P_{B, 0}=0.9$. Особенность процесса заключается в том, что сопротивление к концу разряда возрастает на 5 порядков, значительно превышая сопротивление медной трубки. Такая особенность изменения сопротивления приводит к тому, что основная часть энергии конденсаторной батареи, перешедшая в магнитную энергию трубки, диссипируется на внешнем сопротивлении ко времени $\varpi t \sim 3$ (рис. 6).

Радиусы всех трубок уменьшаются до $\omega t \sim \pi / 2-$ момента максимального сжатия. Затем происходит расширение трубок. Однако если радиусы углеродного стержня и вольфрамовой трубки увеличиваются незначительно, радиус медной трубки из-за теплового взрыва увеличивается резко. При меньших токах в результате 
нагрева тепловое давление становится больше магнитного и медный проводник „медленно“ расширяется. Плотность проводника в процессе расширения сильно неоднородна. Когда внешние части проводника расширятся до характерной плотности, происходит рекомбинация. Начальная степень ионизации в основном холодная $[10,49]$, поэтому при расширении степень ионизации становится практически нулевой, резко возрастает электрическое сопротивление, ток вытесняется из внешних слоев, магнитное давление $(\sim j H)$ резко уменьшается и проводник расширяется с тепловой скоростью. Та часть тока, которая осталась во внешнем слое, из-за резкого роста сопротивления приводит к увеличению джоулевой энергии и возрастанию степени ионизации, увеличению проводимости, однако ток уже не успевает перераспределиться и остановить расширение вещества.

В углеродной, а также в вольфрамовой трубке все функции слабо неоднородны, поэтому временные зависимости плотности, температуры, давления и энергии Гиббса (для углерода) показаны на рис. 6 усредненными по радиусу.

Средняя плотность в углеродной трубке изменяется от начальной плотности графита $\rho=2.25 \mathrm{~g} / \mathrm{cm}^{3}$ до начальной плотности превращения $\rho_{b}=2.97 \mathrm{~g} / \mathrm{cm}^{3}$, затем до плотности $\rho_{c}=3.83 \mathrm{~g} / \mathrm{cm}^{3}$ происходит превращение. На рис. 6 показаны концентрации алмаза $k_{d}$, исходя из двух рассматриваемых здесь моделей: уравнения квазиравновесия (4) и кинетического уравнения (3).

Температура углерода увеличивается незначительно: от начальной $T=300 \mathrm{~K}$ до максимальной $T \approx 400 \mathrm{~K}$, определяемой в основном адиабатическим нагревом. Примерно такую же температуру имеет и вольфрамовая трубка. Разница между ними заметна лишь после стадии превращения. Температура углерода не уменьшается до начальной величины, так как углерод уже имеет плотность алмаза. После превращения графита в алмаз обратного перехода, так называемой графитизации, при столь низкой температуре не происходит. (Характерная температура графитизации $\sim 10^{3} \mathrm{~K}$.) В этом заключается одно из преимуществ рассматриваемого метода изэнтропического превращения. В отличие от ударно-волнового, где температуры достигают значений в зоне превращения $T \sim(1-3) \cdot 10^{3} \mathrm{~K}$ (рис. 5). Следует заметить, что начальную температуру графита можно изменять от гелиевой [7] до очень высокой, что достигается его предварительным джоулевым нагревом в дополнительной электрической цепи. При этом вместо вольфрама можно использовать тугоплавкий прочный диэлектрик.

Температура меди очень неоднородна. Поэтому на рис. 6 показана температура внутренней части и максимальная температура, достигаемая вблизи внешней границы. Если температура внутренней части не превышает $T \sim 450 \mathrm{~K}$, то максимальная температура в момент максимального превращения $T \sim 6 \cdot 10^{3} \mathrm{~K}$, а в конце процесса $T \sim 4 \cdot 10^{4} \mathrm{~K}$.
Радиальные распределения показаны на рис. 7 в момент времени, когда половина графита превратилась в алмаз.

При диффузии магнитного поля наблюдается картина, характерной особенностью которой является распространение волны плотности тока (нелинейного скин-слоя) [50]. Качественная оценка этого явления может быть сделана на основе автомодельного решения. Фронт токовой волны распространяется по закону $x_{f}=\xi_{f}\left(H_{0}^{2} / 8 \pi E_{0}\right)^{\beta / 2}\left(t_{0} \chi_{0}\right)^{1 / 2}\left(t / t_{0}\right)^{\delta}$. Здесь граничное поле $H_{b}=H_{0}\left(t / t_{0}\right)^{\alpha}$; коэффициент диффузии магнитного поля $\chi_{H}=\chi_{H, 0}\left(E / E_{0}\right)^{\beta} ; E-$ энергия на единицу объема, $E_{0}-$ начальное значение энергии. Для автомодельной переменной $\chi$ положение фронта определяется численно: $\xi_{f}=\xi_{f}(\alpha, \beta), \delta=1 / 2+\alpha \beta$. Изменение нелинейного скин-слоя по сравнению с линейным $(\beta=0)$ равно $x_{f} / x_{f, 0}=\left(\xi_{f} / \xi_{f, 0}\right)\left(H_{0}^{2} / 8 \pi E_{0}\right)^{\beta / 2}$ и не зависит от $t$. Величина линейного скин-слоя $x_{f, 0}=\chi_{f, 0}\left(\chi_{0} t\right)^{1 / 2}$, где $\chi_{f, 0} \sim 1$. Ширина фронта токовой волны определяется величиной $\Delta=\chi_{0} / u_{f}$, где $u_{f}=d x_{f} / d t-$ скорость токовой волны. Так как $u_{f} \sim t^{\delta-1}$, то $\Delta \sim \chi_{0} t / x_{f} \sim / x_{f, 0}^{2} / x_{f}$. Величина линейного скин-слоя при $t_{0}=5 \mu \mathrm{s} x_{f, 0} \approx 0.02 \mathrm{~cm}$. Различие величины $x_{f} / R_{0}$ приводит к различным картинам $j(r, t)$. Плотность тока в токовой волне по отношению к однородному распределению тока может возрасти на порядки величин. Это обусловлено вытеснением тока из периферии к центру в результате нагрева вещества и его расширения, отчего значительно уменьшается проводимость вещества. Теплопроводность и излучение играют второстепенную роль.

Магнитное давление формирует волну сжатия, скорость которой определяется величиной магнитного давления и соответственно током. Колебания этих величин обусловлены волнами сжатия и разрежения. Однако при выбранных параметрах и веществах ударные волны не возникают.

Давление на оси $P_{\text {aхis }}$ в квазиравновесии определяется величиной $P_{j H}(r)=0.1 \int_{r}^{R} j H d r$, а среднее давление $P_{a}=\left(1 / R^{2}\right) \times \int_{0}^{R} P(r) 2 r d r$ определяется магнитным давлением $P_{a}=P_{H^{2}}$, где $P_{H^{2}}=H^{2} / 8 \pi$, в общем случае $P_{j H} \approx(1-2) P_{H^{2}}$. Эти соотношения непосредственно следуют из уравнения движения и хорошо выполняются в процессе сжатия при квазиравновесии - рассматриваемых здесь токах и временах. Однако в более быстрых процессах, когда квазиравновесие не успевает установиться, эти соотношения не выполняются.

Влияние теплопроводности по сравнению с джоулевым нагревом в основном незначительно и характеризуется отношением $\chi=\chi_{H} / \chi_{T}$, где $\chi_{T}-$ коэффициент диффузии тепла (электронной температуропроводности), $\chi \sim T$ в области твердой и жидкой фазы, $\chi \sim 1 / T^{4}$ - для плазмы. Лучистая теплопроводность превалирует над электронной теплопроводностью в об- 

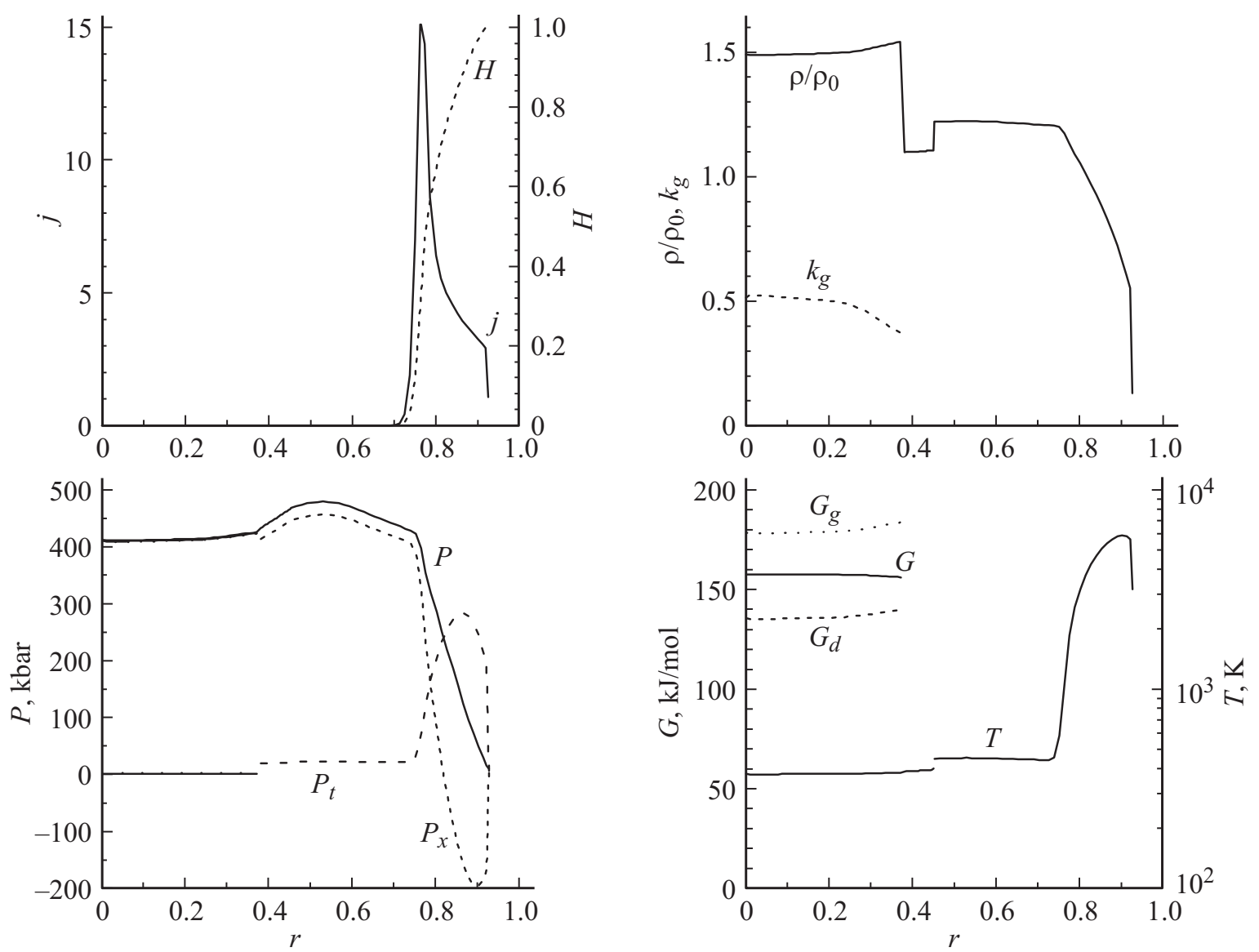

Рис. 7. Радиальные распределения плотности тока, магнитного поля, плотности, концентрации, давления, энергии Гиббса в момент времени, когда половина графита превратилась в алмаз.

ласти высоких температур и низких плотностей в пограничной области. Длина пробега квантов мала, поэтому излучение имеет практически поверхностный характер. Поток энергии на границу обусловлен теплопроводностью, поэтому вблизи внешней границы температура не монотонна, а имеет максимум. Изменение поверхностной температуры определяется теплопроводностью, зависящей от плотности, температуры и степени ионизации. Поэтому в момент, когда они резко изменяются, столь же резко изменяется температура поверхности.

Плотность в углеродной трубке слабо неоднородна $\rho / \rho_{0}=1.50-1.55$. Плотность вольфрама (в промежуточной трубке) изменяется мало из-за его слабой сжимаемости. Плотность меди неоднородна. Внутренняя часть медной трубки поджата до плотности $\rho / \rho_{0} \approx 1.2$, однако вблизи внешней границы плотность резко падает $\rho / \rho_{0} \sim 0.1$.

Температура в углеродной трубке, средней трубке остается низкой и определяется в основном адиабатическим сжатием вещества. Температура во внутренней части медной трубки также не успевает увеличиться, однако во внешней части медной трубки температура, определяемая джоулевым нагревом, имеет характерное значение $\sim 10^{4} \mathrm{~K}$.
Давление (термодинамическое) слабо неоднородно везде, за исключением внешней части медной трубки, где оно уменьшается от значений $P \sim 400-500 \mathrm{kbar}$ до 0. Показаны составляющие давления: упругое $P_{x}$ и тепловое давление $P_{t}$. Внутри волны давление в основном упругое, однако за фронтом токовой волны преобладает тепловое давление.

Энергия Гиббса в графито-алмазной смеси почти однородна. Энергия Гиббса графита больше энергии Гиббса алмаза, поэтому и происходит превращение графита в алмаз. Энергия Гиббса графито-алмазной смеси имеет промежуточное значение.

\section{Заключение}

Впервые метод металлического z-пинча с сохранением сжатого вещества использовался в [8], где изложены результаты работы по переходу красного фосфора в черный. Однако создаваемые там токи были 1 МА и соответственно давление $\sim 50 \mathrm{kbar}$. Параметры выбирались таким образом, чтобы медная трубка вообще не плавилась. Для перехода графит-алмаз давление должно быть на порядок больше и медная трубка обязательно должна взрываться. Это следует из автомодельных со- 
отношений $H^{2} / 8 \pi \approx P$ и $H^{2} / 8 \pi \approx c_{V} \rho T$. Величина тока определяется выбором радиуса трубки и соответственно желаемым размером алмаза. Характерное время сжатия согласуется с динамикой процесса и скоростью токовой волны.

\section{Список литературы}

[1] The Properties of Diamond. / Ed. by J.E. Field. London: Academic Press, 1979. 674 p.

[2] Безруков Г.Н., Бутузов В.П., Самойлович М.И. Алмаз. Киев: Наукова Думка, 1981. 120 с.

[3] Хмельницкий Р.А. // УФН. 2015. Т. 185. № 2. С. 143-159.

[4] Ставер А.М., Губарева Н.В., Лямкин А.Н. // ФГВ. 1984. № 5. C. $100-106$.

[5] Ломоносов И.В., Фортов В.Е., Фролова А.А. и др. // ТВТ. 2005. T. 41. C. $515-521$.

[6] Прут В.В., Храбров В.А., Матвеев В.В., Шибаев С.А. // Письма в ЖЭТФ. 1979. Т. 29. № 1. С. 33-36.

[7] Матвеев В.В., Медведева И.В., Прут В.В. // Письма в ЖЭТФ. 1984. Т. 39. № 5. С. 219-223.

[8] Матвеев В.В., Прут В.В., Храбров В.А. // Письма в ЖТФ. 1978. T. 4. № 9. C. 551-553.

[9] Prut V.V., Shybaev S.A. // J. Phys. D: Appl. Phys. 1996. Vol. 29. P. $3071-3079$.

[10] Прут В.В. // ТВТ. 2005. Т. 43. Вып. 5. С. 713-726.

[11] Blatt F.J. Physics of electronic conduction in solids. Mc GrawHill, 1968. 446 p.

[12] Вихрев В.В., Иванов В.В., Прут В.В. // Физика плазмы. 1986. T. 12. C. $328-335$.

[13] Зельдович Я.Б., Райзер Ю.П. Физика ударных волн и высокотемпературных гидродинамических явлений. М.: Наука, 1966. $688 \mathrm{c}$.

[14] Shapiro S.L., Teukolsky S.A. Black holes, white drafts, and neutron stars. N.Y.: Wiley, 1983. 672 p.

[15] Зельдович Я.Б., Новиков И.Д. Теория тяготения и эволюция звезд. М.: Наука, 1971. 485 с.

[16] Seldin E.J., Nezbeda C.W. // J. Appl. Phys. 1970. Vol. 41. N 8. P. 3389-3395.

[17] Nicklow R., Wakabayashi N., Smith H.G. // Phys. Rev. B. 1972. Vol. 5. P. 4951-4956.

[18] Gauster W.B., Fritz J.J. // J. Appl. Phys. 1974. Vol. 45. N 8. P. 3309-3313.

[19] Zhao Y.X., Spain I.L. // Phys. Rev. B. 1989. Vol. 40. N 2. P. 993-997.

[20] Hanfland M., Beister H., Syassen K. // Phys. Rev. B. 1989. Vol. 39. P. 12598-12603.

[21] Yagi T., Utsumi W., Yamakata M. et al. // Phys. Rev. B. 1992. Vol. 46. P. 6031-6037.

[22] Alder B.J., Christian R.H. // Phys. Rev. Lett. 1961. Vol. 7. P. 367-370.

[23] Doran D.J. // J. Appl. Phys. 1963. Vol. 34. P. 844-848.

[24] Coleburn N.L. // J. Chem. Phys. 1964. Vol. 40. P. 71-76.

[25] Gust W.H. // Phys. Rev. B. 1980. Vol. 22. P. 4744-4749.

[26] Morris D.G. // J. Appl. Phys. 1980. Vol. 51. P. 2059-2065.

[27] Nellis W.J., Mitchell A., Mc Mahan A.K. // J. Appl. Phys. 2001. Vol. 90. P. 696-702.

[28] Yin T., Cohen M.L. // Phys. Rev. B. 1984. Vol. 29. N 12. P. 6996-7002.

[29] Fahy S., Louie S.G., Cohen M.L. // Phys. Rev. B. 1987. Vol. 35. P. 7623-7628.
[30] Jansen H.J.F., Freeman A.J. // Phys. Rev. B. 1987. Vol. 35. P. 8207-8213.

[31] Boettger J.C. // Phys. Rev. B. 1997. Vol. 55. P. 11202-11207.

[32] Жарков В.Н., Калинин В.А. Уравнения состояния твердых тел при высоких давлениях и температурах. М.: Наука, 1968. 312 c.

[33] Vinet P., Ferrante J., Smith J.R., Rose J.H. // J. Phys. C: Sol. Stat. Phys. 1986. Vol. 19. P. L467-L473.

[34] Mc Skimin H.J., Andreatch P. // J. Appl. Phys. 1972. Vol. 43. P. 2944-2949.

[35] Grimsditch M.H., Ramdas A.K. // Phys. Rev. B. 1975. Vol. 11. P. 3139-3145.

[36] Vogelgesang R., Ramdas A.K., Rodriguez S. et al. // Phys. Rev. B. 1996. Vol. 54. P. 3989-3995.

[37] Zouboulis E.S., Grimsditch M., Ramdas A.K., Rodriguez S. // Phys. Rev. B. 1998. Vol. 57. P. 2889-2895.

[38] Gillet Ph., Fiquet G., Daniel I., Reynard B., Hanfland M. // Phys. Rev. B. 1999. Vol. 60. P. 14660-14667.

[39] Павловский М.Н. // ФТТ. 1971. Т. 13. Вып. 3. С. 893-897.

[40] Павловский М.Н., Дракин В.П. // Письма в ЖЭТФ. 1966. T. 4. № 5. С. $198-202$.

[41] Александров И.В., Гончаров А.Ф., Зисман А.И., Стишов С.М. // ЖЭТФ. 1987. Т. 93. № 2(8). С. 680-685.

[42] Nielsen O.H. // Phys. Rev. B. 1986. Vol. 34. P. 5808-5813.

[43] Xie J., Chen S.P., Tse J.S., Gironcoli S., Baroni S. // Phys. Rev. B. 1999. Vol. 60. P. 9444-9449.

[44] Herrero C.P., Ramírez R. // Phys. Rev. B. 2000. Vol. 63. P. $024103-024107$.

[45] Kunc K., Loa I., Syassen K. // Phys. Rev. B. 2003. Vol. 68. P. 094107-094112.

[46] Mounet N., Marzari N. // Phys. Rev. B. 2005. Vol. 71. P. 205214-205219.

[47] Maezono R., Ma A., Towler M.D., Needs R.J. // Phys. Rev. Lett. 2007. Vol. 98. P. 025701-025706.

[48] Калиткин Н.Н. // ФТТ. 1965. Т. 7. Вып. 2. С. 355-359.

[49] Прут В.В. // ЖТФ. 2004. Т. 74. Вып. 12. С. 10-20.

[50] Шнеерсон Г.А. // ЖТФ. 1973. Т. 43. Вып. 3. С. 419-428. 